Marc Hooghe, Dietlind Stolle, Valérie-Anne Mahéo \& Sara Vissers

\title{
Why can't a Student be more like an Average Person? Sampling and Attrition Effects in Social Science Field and Laboratory Experiments
}

\section{Annals of the American Academy of Political and Social Sciences, 628(1), 2010, 85-96.}

(C) Sage Publications, Thousand Oaks 2010. DOI: 10.1177/0002716209351516

\section{Introduction}

The majority of experimental laboratory research in the social sciences is based on convenience samples. Most of these studies recruit among undergraduate university students. Undergraduates are a convenient target population for researchers as course credits are often used to render students' participation compulsory. The use of convenience samples and the overrepresentation of undergraduate students have raised some concerns about the external validity of experimental results (Kam et al. 2007; Levitt \& List 2007). Although there might be several research questions for which a student sample is appropriate, research has found that concerns remain about the generalizability of the results to off-campus populations. Moreover, to answer some specific research questions might simply require the inclusion of non-student populations. 
In this essay, we make a case for the inclusion of non-student samples in field and laboratory experiments wherever the research question requires it. We particularly focus on the recruitment of people with lower socio-economic background characteristics, a group that is largely under-represented in most research and particularly in experimental projects. In addition, we show that the inclusion of non-student samples can affect the results and implications of the research. Yet our experience shows that recruitment among non-student groups creates new methodological challenges (i.e., recruitment strategy, setting of the experiments, random assignment, incentives, attrition) that are of interest for researchers who intend to include non-students in their experimental design.

The inclusion of non-student samples can affect the results and implications of the research. Yet our experience shows that recruitment among non-student groups creates new methodological challenges.

An associated problem for experimental researchers is that of attrition of participants. Different populations, e.g. student and non-students might result in different drop out rates, which in turn requires different strategies to maintain the motivation of participants. Long-term exposure to experimental manipulations is often rendered difficult because of the tendency of specific groups within the population to drop out of the experiment. As a result, researchers tend to concentrate on effects of a relatively brief exposure, but these can be practically and theoretically less relevant. Retaining participants in a long-lasting experiment, however, is at 
least as serious a challenge as recruiting a non-compulsory audience for the experiments in the first place.

(...)

\section{Limitations of Student Samples: Research on Youth Mobilization}

In addition to all the doubts about the exclusive use of student samples, there are obviously specific research questions that require non-student sampling. An example from our own research experience will make this point more clear. We are interested in the growing concern about declining turnout across established democracies. Much of this decline can be explained by generational replacement. The problem is that this generational effect is most visible among the young and the socially under-privileged groups such as the unemployed (Bennulf \& Hedberg 1999; Hooghe \& Stolle 2004; Tam Cho, Gimpel \& Wu 2006; McDewitt \& Kiousis 2006). For example, since the 1993 Canadian federal election, turnout in the youngest generation has dropped over 30 points among high school dropouts and 15 points or more among those who have completed high school and/or some college, but it has held steady among young university graduates. Similarly, in Europe, young socio-economically disadvantaged citizens with low levels of education or unemployment are disproportionately turning out less to the polls (Bennulf \& Hedberg 1999). Clearly, efforts to foster political engagement need to target young people who are not college bound (Pasek et al. 2006). 
Field and laboratory experiments are vital here because they make it possible to isolate the effects of different ways of mobilizing young people, free of other confounding factors. Some of the few experiments that have been conducted do not use a full experimental design including pre- and post-test and the inclusion of a control group (Beaumount et al. 2006; Bers and Chou 2006), or do not study long-term effects (Pinkleton 1998; Addonizio 2004; Hansmann et al. 2005). One of the few exceptions is the quasi-experimental study with randomly assigned students by Bernstein and Meizlish (2003), which includes a longitudinal analysis three years after the treatment and the study by Phillips (2004) with mobilizational treatments of high school juniors. Most importantly though, these studies have typically focused either on high school or on university students (Bernstein and Meizlish 2003; Hansmann et al. 2005; Livingston and Kidder 1973; Niemi and Junn 1998; Smith 1999). As a result, the policy prescriptions that emerge do not adequately address the alarming decline in political participation on the part of those who are leaving school with only a grade 9 or grade 10 education, or those who come from socio-economically disadvantaged backgrounds or who are young unemployed. Moreover, many strategies that are effective for university-bound adolescents may not work for young people who lack equivalent literacy and other political skills (Nickerson 2006; Tedesco 2007). The theoretical issue and policy puzzle here then is about finding generalizable political mobilization strategies that work for young people but which at the same time allow us to address the specific circumstances and needs of populations from a lower socio-economic background.

In our experiments then we examined the comparative mobilizational potential of face-to-face versus online campaigns developed around the themes of climate change. Because we expect differential results from university students and non college bound youth, we sampled 
participants from high and low socio-economic status backgrounds. All of the roughly 400 participants, between the ages of 18 to 25 were exposed to the same mobilization content, besides the control group. The non college bound population was reached in three different ways in disadvantaged neighborhoods: vocational/professional schools, work and employment programs, and continuing education schools. All participants were exposed to one manipulation tool, and exposed to a pre-, and two post-tests (immediately after the manipulation and after three months following the experimental condition).

\section{(...)}

\section{Attrition}

Using a broader recruitment pool would ensure a more diverse sample of participants at the outset of an experiment, but still this offers no guarantee that this level of diversity can be maintained throughout the experiment as some groups have a stronger propensity to drop out than other groups. These differential attrition levels might endanger the validity of the results of especially long-term effect experiments. Attrition is a well-known problem in long-term panel surveys, but also in experimental designs. This time factor in long term experiments raises an extra problem which does not exist in classical experiments, in which subjects only have to participate once (Housman \& Wise 1979). Attrition occurs when subjects drop out of the experimental study, for instance after the pre-test or after the first post-test. This can have several causes, which may have consequences for the internal validity of the experiment and the interpretation of the results. Attrition by itself does not invalidate experimental results (Cook \& Campbell 1979, Fitzgerald, Gottschalk and Moffitt 1998). Attrition is only a serious 
problem when it is non-random, because only then it may erode the initial experimental design and the random assignment across the treatment groups (Housman \& Wise 1979). If participants drop out of the study for reasons that are not related to the subject of the study, it is very likely that attrition will be less of a threat to internal validity. For instance, when a subject does not show up for the second experimental session or does not answer follow-up questionnaires because of medical reasons, we might still assume that this condition might be randomly spread across the sample. It might also be that the researchers lost track of the participant and that they are not able to contact them anymore, e.g. due to the fact that the participant moved. However, if attrition is in some way related to the (in)dependent variable or the experimental treatment, this poses a serious challenge for the validity of the experimental results. Therefore it is of crucial importance to test intra-treatment attrition, i.e. to compare the drop-out rates across the different treatment groups, including the control group; and in the past some statistical techniques have been developed to counter the effects of non-random attrition (Horowitz \& Manski 2000). For instance, attrition might be higher among participants who were not interested in the subject in the first place, or to the contrary, when they are not triggered sufficiently. Returning to the example of the mobilization experiments we could expect higher attrition rates among the two extreme participant groups: the respondents for whom global warming was not a topic of great concern on the one hand, and the respondents who were initially already very interested and engaged in the environmental issue on the other hand. If a person is very knowledgeable about the substantive mobilization topic this might have a negative effect on the motivation to continue to participate in the experiment as he or she is not triggered sufficiently by the information provided during the experimental condition. In our experiments, for the respondents with less formal education we found indeed a significant negative relation between self-indicated understanding of global warming in the 
pre-test and attrition. Another expectation might be that there would be attrition differences between the treatment and the control groups, as the latter were exposed to a treatment that normally does not make sense. In our own experiment, however, we did not encounter any significant differences in attrition rate between control and treatment groups. In some experiments, attrition bias can be countered by relying on independently collected information about behavioral outcomes of the experiment (e.g., official registers about whether a citizen participated in voting or not).

Moreover it is also important to be aware of possible attrition effects as a result of the different treatments. For instance, participants with lower levels of internet skills might be more likely to drop out of the experimental groups where the mobilization is internet mediated. The same mechanism could be at stake for individuals with lower levels of civic skills in the interactive role play treatment group. One important aspect that we would like to address here is that one should be extremely careful when different groups of respondents are included in the experiment, for instance students and non-students. Students and non-students have different characteristics, and this implies they will need different incentives to stay in all three waves of the study. Our experiments suggest that attrition is substantially higher for the lower socioeconomic population. We can observe a substantial difference in the drop-out rates between the student and non-student population from the first wave to the second wave, but especially in the long-term, these differences seem to become most apparent. Financial incentives and other incentives, may reduce this problem to some extent. In order to boost the response rates of the non-student population for the third wave, we organized extra reminder calls and extra financial incentives between the second and the third wave. Especially in long-term exposure experiments, and experiments with different target populations, (non-randomized) attrition is a 
serious challenge. This is probably one of the many reasons why long term exposure experiments are so rare in social sciences where the financial resources to conduct experimental studies are very limited in comparison with the biomedical sciences. However, we would like to defend the necessity of more experiments in the long run. In situations where money is a scarce good, researchers should be creative in developing other incentives to motivate their participants in order to conduct experiments on the effects of long-term exposure.

The results we obtain with our standard convenience samples of undergraduate students simply cannot be generalized toward other population groups. Whenever possible or appropriate, it is indeed important to move beyond the 'narrow base' of undergraduate participants.

\section{Conclusion}

In recent years, there has been a call within medical research to diversify the participants of medical experiments. The main reason is that some effects might be found, e.g., among white males, but that there is no reason to assume that the new experimental drug will be just as effective, e.g., among Afro-Americans, Asians or women. For some specific drugs trials, pharmaceutical companies now increasingly rely on diverse populations (Epstein 2007). Basically, our call is to venture into the same direction with social science experiments. In various cases, the results we obtain with our standard convenience samples of undergraduate students simply cannot be generalized toward other population groups. Whenever possible or appropriate, it is indeed important to move beyond the 'narrow base' of undergraduate 
participants. This might be a serious challenge for conducting the field work, but this broadening might lead to more reliable results from the experiments being conducted.

Recently, List (2008) has called for efforts to arrive at more robust findings from experimental research by combining different research strategies. Given all the caveats associated with experimental research, it would be unwise to arrive at firm conclusions, based on the results of just one research design. Based on our own experience, therefore, we agree with List, in arguing for mixed-method research efforts, combining various settings and methods in order to arrive at more reliable findings. While research on undergraduate students might be a very good start for various research questions, we also have to keep in mind that undergraduate students are not average citizens, and that they might have specific characteristics that limit the possibility to generalize findings on this group toward the whole population. Self-evidently, it still makes sense to use specific student samples as a first step in order to determine the occurrence of a causal relation, or to study a phenomenon that is limited to this group. But when we want to study a broader social phenomenon or specific effects in other population groups outside the university campus, clearly student samples can tell us only part of the story. Efforts to corroborate experimental findings - e.g., with results from survey research, or from specific small experiments among different target groups - might be used to strengthen the validity of our findings. 